\title{
Sudden cardiac death in asymptomatic patients with aortic stenosis
}

\author{
Jan Minners 이, ${ }^{1}$ Anne Rossebo, ${ }^{2}$ John B Chambers 지, ${ }^{3}$ Christa Gohlke-Baerwolf, ${ }^{1}$ \\ Franz-Josef Neumann, ${ }^{1}$ Kristian Wachtell, ${ }^{2}$ Nikolaus Jander ${ }^{1}$
}

- Additional material is published online only. To view, please visit the journal online (http://dx.doi.org/10.1136/ heartjnl-2019-316493).

${ }^{1}$ Department of Cardiology and Angiology II, University Heart Center Freiburg / Bad Krozingen, Bad Krozingen, Germany ${ }^{2}$ Department of Cardiology, Oslo Universitetssykehus Rikshospitalet, Oslo, Norway ${ }^{3}$ St Thomas Hospital, London, UK

\section{Correspondence to}

Dr Jan Minners, Department of Cardiology and Angiology II, University Heart Center Freiburg / Bad Krozingen, Bad Krozingen, Germany;

Jan.Minners@universitaetsherzzentrum.de

Received 27 January 2020 Revised 15 June 2020 Accepted 24 June 2020 Published Online First 31 July 2020

\begin{abstract}
Objective We retrospectively analysed outcome data from the Simvastatin and Ezetimibe in Aortic Stenosis (SEAS) study to assess the incidence and potential risk factors of sudden cardiac death (SCD) in this prospectively followed cohort of asymptomatic patients with aortic stenosis (AS).
\end{abstract}

Methods Of the 1873 patients included in SEAS, 1849 $(99 \%)$ with mild to moderate AS (jet velocity $2.5-4.0 \mathrm{~m} / \mathrm{s}$ at baseline) and available clinical, echocardiographic and follow-up data were analysed. Patients undergoing aortic valve replacement were censored at the time of operation.

Results During an overall follow-up of $46.1 \pm 14.6$ months, SCD occurred in 27 asymptomatic patients $(1.5 \%)$ after a mean of $28.3 \pm 16.6$ months. The annualised event rate was $0.39 \% / y e a r$. The last follow-up echocardiography prior to the event showed mild to moderate stenosis in 22 and severe stenosis (jet velocity $>4 \mathrm{~m} / \mathrm{s}$ ) in 5 victims of SCD. The annualised event rate after the diagnosis of severe stenosis was $0.60 \%$ /year compared with $0.46 \% / y e a r$ in patients who did not progress to severe stenosis $(p=0.79)$. Patients with $S C D$ were older $(p=0.01)$, had a higher left ventricular mass index (LVMI, $\mathrm{p}=0.001$ ) and had a lower body mass index (BMI, $p=0.02$ ) compared with patients surviving followup. Cox regression analysis identified age (HR 1.06, $95 \% \mathrm{Cl} 1.01$ to 1.11 per year, $p=0.02)$, increased LVMI (HR 1.20, 95\% Cl 1.10 to 1.32 per $10 \mathrm{~g} / \mathrm{m}^{2}, \mathrm{p}<0.001$ ) and lower BMI (HR 0.87, 95\% Cl 0.79 to 0.97 per kg/ $\left.\mathrm{m}^{2}, \mathrm{p}=0.01\right)$ as independent risk factors of SCD. Conclusion SCD in patients with asymptomatic mild to moderate $A S$ is rare and strongly related to left ventricular hypertrophy but not stenosis severity.

\section{INTRODUCTION}

SLinked

- http://dx.doi.org/10.1136/ heartjnl-2020-317339

Check for updates

(C) Author(s) (or their employer(s)) 2020. No commercial re-use. See rights and permissions. Published by BMJ.

To cite: Minners J,

Rossebo A, Chambers JB

et al. Heart

2020;106:1646-1650
Aortic valve stenosis is a progressive disease affecting around $3 \%$ of adults aged $\geq 75$ years in the western world, and its prevalence is expected to increase further with an ageing population. ${ }^{1-3}$ The overall survival of patients with aortic stenosis (AS) during the initial extended asymptomatic period has been considered comparable to age-matched controls. However, sudden cardiac death (SCD) is a significant concern in these patients, with a reported incidence ranging from $0.3 \% /$ year to $3.1 \% / y e a r$, possibly depending on AS severity as well as a variety of non-valve-related factors, including prior myocardial infarction (MI) and haemodialysis. ${ }^{4-9}$

The Simvastatin and Ezetimibe in Aortic Stenosis (SEAS) study was a randomised, double-blind, clinical trial of 1873 patients aged 45-85 years with asymptomatic mild-to-moderate AS with peak aortic-jet velocity $2.5-4.0 \mathrm{~m} / \mathrm{s}$ and normal left ventricular (LV) ejection fraction. ${ }^{10}{ }^{11}$ Importantly, extensive exclusion criteria (eg, concomitant atherosclerotic diseases in need of lipid-lowering therapy, known coronary artery disease, known cerebrovascular disease, diabetes mellitus, more than mild renal and liver failure and severe obstructive lung disease) served to obtain a study population virtually free from comorbidities allowing for the targeted assessment of the impact of valvular disease on the incidence of SCD.

We, therefore, retrospectively analysed outcome data from the SEAS study with the aim to assess the incidence and potential risk factors of SCD in this prospectively followed, low-risk cohort of asymptomatic patients with AS.

\section{METHODS}

Study design and results of the SEAS study have been published previously. ${ }^{10} 11$ Patients were followed up at 6-month intervals in cardiology outpatient clinics with clinical evaluation and fasting blood samples, as well as annual electrocardiographic and echocardiographic evaluation. In the individual patient, the decision to proceed to valve replacement was made by the treating physician according to the recommendations set out in the then-current guidelines. The SEAS study showed no effect of intensive lipidlowering therapy with simvastatin and ezetimibe versus placebo on the primary composite cardiovascular endpoint (including aortic valve replacement (AVR), cardiovascular death, heart failure as a result of the progression of AS and ischaemic endpoints, including revascularisation, $\mathrm{MI} /$ unstable angina and non-haemorrhagic stroke) and no effect on the key secondary valve-related endpoints (AVR, heart failure or cardiovascular death) and echocardiographic progression of AS. ${ }^{10}$

\section{Echocardiography}

Echocardiograms were obtained at baseline and yearly after that until completion of follow-up or valve surgery and were submitted to the study Echocardiography Core Laboratory for central analysis. Readers were blinded for treatment and sequence, according to a predefined standardised echocardiographic study protocol. ${ }^{11}$ AS severity was measured according to accepted guidelines, and LV mass was calculated according to the Devereux formula and indexed for body surface area. ${ }^{12}{ }^{13} \mathrm{Left}$ 
ventricular hypertrophy was defined as left ventricular mass index (LVMI) $>95 \mathrm{~g} / \mathrm{m}^{2}$ in women and $>115 \mathrm{~g} / \mathrm{m}^{2}$ in men.

\section{Study endpoints}

The main study endpoints have been described previously. ${ }^{10}{ }^{11}$ All endpoints were predefined by an endpoint classification protocol, classified by an independent Endpoint Classification Committee (ECC) of two experienced cardiologists blinded to treatment in the main study. Any classification disagreements were settled through adjudication meetings. All deaths in the study $(\mathrm{n}=205)$ were reported from the local study sites as potential endpoints and classified by the ECC as cardiovascular $(n=103)$ or noncardiovascular events. The cardiovascular death endpoint, by definition, included death from complications of MI, progressive heart failure, cerebrovascular disease, complications of cardiac surgery or intervention, and other cardiac or cardiovascular diseases. Witnessed sudden death and non-witnessed unexpected death were classified as cardiovascular death unless other causes of death could be documented with reasonable certainty (eg, excluding patients with known signs or symptoms of other fatal diseases when last observed).

\section{SCD population}

SCD was a predefined subset of the cardiac death endpoint, specified as instantaneous cardiac death, non-witnessed unexpected death and cardiac death occurring $<24$ hours after onset of new cardiac symptoms. In the SEAS study, 38 patients with available baseline echocardiographic data experienced a fatal event classified as SCD. Of these, 27 patients had a so far uneventful follow-up (without documented signs or symptoms of a serious event that could have lent the treating physician to suspect an increased risk of SCD) and formed the basis of the present analysis (for details, see online supplementary table 1). The remaining 11 patients classified as having died from SCD during the SEAS study had either developed cardiac signs or symptoms and an indication for AVR $(n=7)$ or died after AVR was performed $(n=4)$ and were consequently excluded from the analysis (online supplementary table 2). A total of 1822 patients who did not suffer SCD served as comparisons with patients undergoing AVR censored at the time of operation (figure 1).

\section{Statistical analysis}

Statistical analysis was performed using SPSS V.23. All categorical data are presented as percentages and all continuous data as mean values $\pm S D$. Cox regression analysis was performed to evaluate variables for the prediction of SCD, and HRs with corresponding $95 \%$ CIs were calculated. Variables with a p value

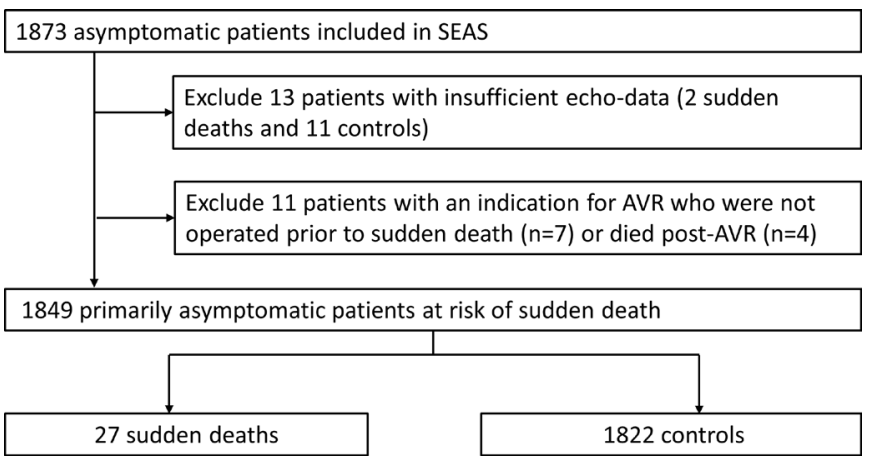

Figure 1 Study population of the seas trial. AVR, aortic valve replacement; SEAS, Simvastatin and Ezetimibe in Aortic Stenosis. of $<0.05$ in univariate analysis were included in the multivariate model, which consequently consisted of three variables (age, body mass index (BMI) and LVMI). There were 0, 2 and 30 missing values, respectively. Missing values were imputed for Cox regression analysis. A two-tailed $\mathrm{p}<0.05$ was considered statistically significant.

The present study was performed without patient involvement. Patients were not invited to comment on the study design and were not consulted to develop patient-relevant outcomes or to interpret the results. Patients were not invited to contribute to the writing or editing of this document for readability or accuracy.

\section{RESULTS}

During a mean follow-up of $46.1 \pm 14.6$ months, covering a total of 7100 patient-years of follow-up, SCD occurred in 27/1849 $(1.5 \%)$ asymptomatic patients after a mean of $28.3 \pm 16.6$ months. The annualised event rate was $0.39 \% /$ patient-year of follow-up, similar to the $0.29 \% /$ year observed in the general population. ${ }^{14}$ Table 1 provides baseline clinical and echocardiographic characteristics of the study population. Patients with SCD were older $(p=0.01)$, had a higher LVMI $(p=0.001)$ and had a lower BMI $(p=0.02)$ compared with those surviving follow-up. Randomisation to either simvastatin/ezetimibe or placebo in the original SEAS study had no impact on the occurrence of SCD. There was no significant difference in baseline echocardiographic parameters of stenosis severity between groups (jet velocity $\mathrm{p}=0.695$, mean gradient $\mathrm{p}=0.678$, aortic valve area (AVA) $\mathrm{p}=0.338$, $\mathrm{AVA}_{\text {index }} \mathrm{p}=0.821$ ). Cox regression analysis identified age (HR $1.06,95 \%$ CI 1.01 to 1.11 per year, $\mathrm{p}=0.02$ ), increased LVMI (HR 1.20, 95\% CI 1.10 to 1.32 per $10 \mathrm{~g} / \mathrm{m}^{2}, \mathrm{p}<0.001$ ) and lower BMI (HR $0.87,95 \%$ CI 0.78 to $0.97 \mathrm{per} \mathrm{kg} / \mathrm{m}^{2}, \mathrm{p}=0.01$ ) as independent risk factors of SCD (figure 2 and table 2). Comparable results were obtained when restricting the analysis to patients with moderate stenosis at baseline (jet velocity $>3 \mathrm{~m} / \mathrm{s}$, online supplementary tables 3 and 4).

\section{Patients with severe stenosis}

Results in the subgroup of patients who, at any time during follow-up, were diagnosed with severe AS (based on jet velocity $>4 \mathrm{~m} / \mathrm{s}, \mathrm{n}=650$, overall follow-up after the diagnosis of severe stenosis $15.3 \pm 16.0$ months) mirrored the findings in the whole cohort. Five patients $(0.8 \%)$ suffered an SCD with a mean of $14.4 \pm 8.4$ months after the initial diagnosis of severe stenosis. The annualised event rate after the diagnosis of severe stenosis was $0.60 \% /$ year as compared with $0.46 \% /$ year in patients with mild to moderate stenosis $(p=0.79)$. A meaningful Cox regression analysis was precluded by small patient numbers in the SCD group.

\section{Stenosis severity over time}

Data to assess the development of AS severity over time ( $>1$ reading for jet velocity) were available in 1765 patients $(96 \%$ of the study population). Maximum jet velocity increase over the entire follow-up ( $46.1 \pm 14.6$ months) was $0.52 \pm 0.66 \mathrm{~m} / \mathrm{s}$ in SCD and $0.62 \pm 0.60 \mathrm{~m} / \mathrm{s}$ in those surviving follow-up $(\mathrm{p}=0.496)$, and increases per year were $0.16 \pm 0.41$ and $0.17 \pm 0.52 \mathrm{~m} / \mathrm{s}$, respectively $(\mathrm{p}=0.876)$. No patient reached the very severe AS range (jet velocity $>5.0 \mathrm{~m} / \mathrm{sec}$ ). AVA was available in 579 of the 650 patients who were diagnosed with severe stenosis based on the jet velocity criterion. It was $1.10 \pm 0.36 \mathrm{~cm}^{2}$ at baseline and $0.89 \pm 0.30 \mathrm{~cm}^{2}$ when severe stenosis was diagnosed. In patients with SCD with a follow-up of $>2$ years $(n=7)$, we found no 


\begin{tabular}{|c|c|c|c|}
\hline & Sudden death & Controls & \\
\hline & $(n=27)$ & $(n=1822)$ & $P$ value \\
\hline Age (years) & $72 \pm 8$ & $67 \pm 10$ & 0.01 \\
\hline Female (\%) & 26 & 39 & 0.231 \\
\hline Body mass index $\left(\mathrm{kg} / \mathrm{m}^{2}\right)$ & $25.0 \pm 4.3$ & $26.9 \pm 4.4$ & 0.024 \\
\hline Blood pressure diastolic (mm Hg) & $83 \pm 11$ & $82 \pm 10$ & 0.642 \\
\hline Blood pressure systolic (mm Hg) & $148 \pm 21$ & $145 \pm 20$ & 0.376 \\
\hline Hypertension (\%) & 51 & 59 & 0.442 \\
\hline Pulse rate (beats/min) & $69 \pm 10$ & $68 \pm 11$ & 0.681 \\
\hline Patients on simvastatin/ezetimibe (\%) & 52 & 50 & 0.998 \\
\hline Ejection fraction (\%) & $67 \pm 6$ & $66 \pm 7$ & 0.873 \\
\hline LV end diastolic diameter (mm) & $5.1 \pm 0.8$ & $5.0 \pm 0.6$ & 0.714 \\
\hline LV end systolic diameter (mm) & $3.2 \pm 0.6$ & $3.2 \pm 0.6$ & 0.836 \\
\hline LVMI (g/m²) & $122 \pm 49$ & $101 \pm 31$ & 0.001 \\
\hline Jet velocity $(\mathrm{m} / \mathrm{s})$ & $3.1 \pm 0.5$ & $3.1 \pm 0.5$ & 0.695 \\
\hline Increase peak velocity $(\mathrm{m} / \mathrm{s})^{*}$ & $0.52 \pm 0.66$ & $0.62 \pm 0.60$ & 0.496 \\
\hline Jet velocity $>3.0 \mathrm{~m} / \mathrm{s}(\%)$ & 51 & 48 & 0.699 \\
\hline Mean pressure gradient (mm Hg) & $22 \pm 8$ & $23 \pm 9$ & 0.678 \\
\hline Aortic valve area $\left(\mathrm{cm}^{2}\right)$ & $1.2 \pm 0.5$ & $1.3 \pm 0.5$ & 0.338 \\
\hline Creatinine (micromol/L) & $95 \pm 15$ & $94 \pm 16$ & 0.636 \\
\hline Haemoglobin (g/L) & $137 \pm 16$ & $139 \pm 12$ & 0.383 \\
\hline Glucose (mmol/L) & $5.3 \pm 1.0$ & $5.3 \pm 0.8$ & 0.809 \\
\hline
\end{tabular}

$\mathrm{LV}$, left ventricular; LVMI, left ventricular mass index.

indication that SCD was preceded by a sudden acceleration of stenosis severity prior to the event with a mean yearly increase in jet velocity of $0.23,0.24$ and $0.29 \mathrm{~m} / \mathrm{s}$ after 1,2 and 3 years, respectively.

\section{Operative mortality}

Operative mortality in SEAS was 2.6\% (14 of 545 patients in whom AVR was performed), amounting to the eightfold of the yearly SCD risk in the entire population. Overall postoperative follow-up was $21.8 \pm 14.8$ months, during which four additional patients with an initially uneventfully recovery from surgery died from SCD a mean of $17.7 \pm 20.7$ months after AVR (annualised event rate $0.42 \% /$ year).

\section{DISCUSSION}

The main finding of the current analysis demonstrates that the incidence of SCD in low-risk patients with asymptomatic AS is comparable to the general population, suggesting that aortic valve disease per se is not a risk factor for SCD.

\section{Incidence of SCD}

The incidence of SCD in the general population is estimated at $0.1 \% /$ year, with major geographical differences and a clear agerelated increase. ${ }^{15}$ For instance, in the population-based Atherosclerosis Risk in Communities (ARIC) study, participants (mean age 72 years) who were free from cardiovascular disease at baseline showed an annualised event rate of SCD of 0.29\%/year. ${ }^{14}$ In asymptomatic patients with AS, the risk of SCD has generally been reported at $<1 \% /$ year. ${ }^{91617}$ However, recent publications of data from a large registry have reported a yearly incidence of $1.4 \%,{ }^{45}$ suggesting that against current beliefs, SCD has to be considered a significant threat even in asymptomatic patients. Consequently, the purported increased risk of SCD was repeatedly brought forward in support of early valve replacement in asymptomatic patients with AS. ${ }^{18} 19$

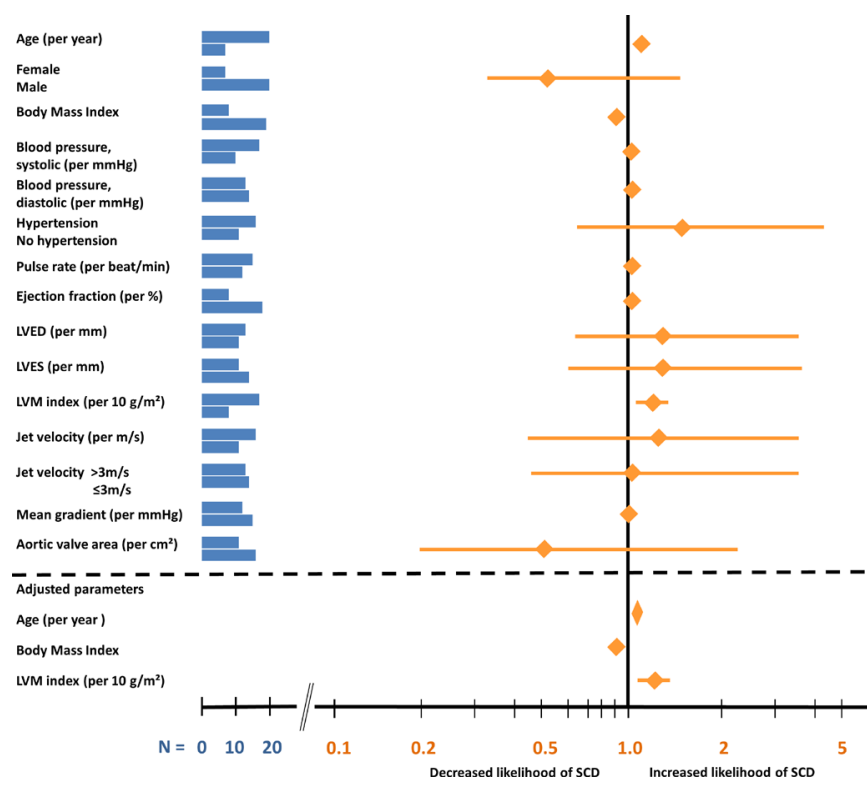

Figure 2 Univariate (top) and multivariate (bottom) Cox regression analyses for SCD during $46.1 \pm 14.6$ months of follow-up in the Simvastatin and Ezetimibe in Aortic Stenosis study. The number of events for each variable is reflected by the dark, horizontal bars with separation at the median for continuous variables. A forest plot visualisation of HRs for SCD is provided on the right. LVM, left ventricular mass; SCD, Sudden cardiac death; LVED, left ventricular enddiastolic diameter; LVES, left ventricular endsystolic diameter.

\section{Risk factors for SCD}

However, published risk factors for SCD in patients with AS are numerous and span the spectrum from valve-related via cardiac to non-valve-related. The risk of SCD associated with haemodialysis, as an example of the latter, is increased by a factor of almost $4^{4}$ but is unlikely to be influenced by valve intervention. Therefore, the SEAS trial, which included patients with mild to moderate AS at baseline with a low comorbidity burden (both cardiovascular as well as non-cardiovascular) and without any known other risk factors for SCD (except for hypertension) provides an ideal dataset to dissect out the specific impact of aortic valve pathology on the incidence of SCD in AS. The data clearly demonstrate that SCD is rare in asymptomatic patients with AS who do not have non-valve-related risk factors. As a consequence, valve replacement is unlikely to reduce the risk of SCD in patients with AS in general. This finding is further supported by taking perioperative mortality and postoperative risk of SCD into consideration, which proved substantially higher than the baseline risk of SCD in the current analysis. Furthermore, sporadic evidence from postmortems demonstrates a high prevalence of undiagnosed coronary artery disease in patients with SCD (online supplementary table 1) despite the known risk factors for coronary artery disease representing exclusion criteria in the SEAS study. Once symptoms develop, prompt valve replacement appears advisable as exemplified by the seven patients from SEAS who died from SCD awaiting AVR. LV hypertrophy was associated with SCD in multivariate analysis, a finding adding to the evidence that LV hypertrophy is associated with clinical events at all stages of AS. ${ }^{20-23}$ In addition, in the general population, LV hypertrophy was one of the strongest predictors of SCD. ${ }^{24}$ Age interacts with LV hypertrophy in hypertensive patients with LV hypertrophy. Older age leaves more time for disease burden, and thus, more residual 
Table 2 Risk of sudden cardiac death-Cox regression

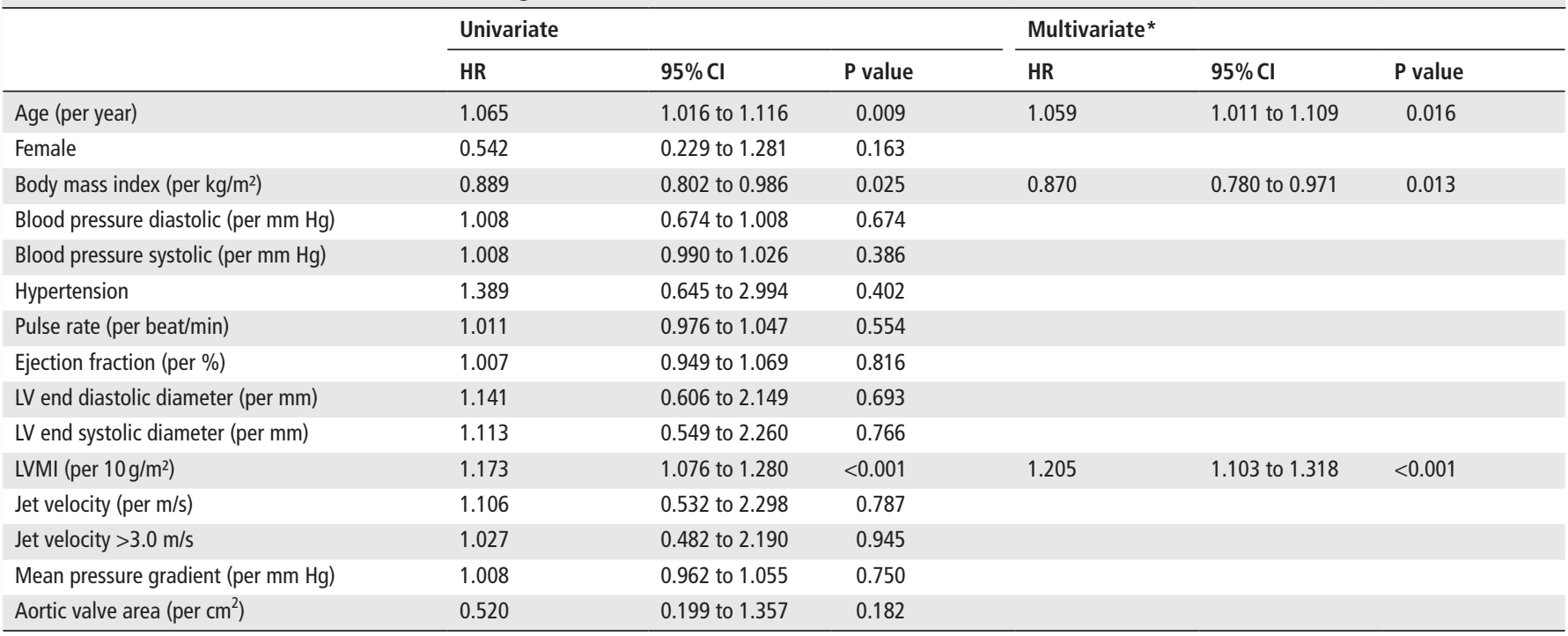

*Variables with $p<0.05$ on univariate were included in the multivariate analysis.

LV, left ventricular; LVMI, left ventricular mass index.

hypertrophy is prevalent in older patients as a consequence of higher initial LV mass. ${ }^{25}$ Our finding that age is an independent predictor of SCD of approximately $6 \%$ per 1 year suggests that there is some residual risk associated with age, similar to the $7 \%$ seen in hypertensive patients. ${ }^{25}$ In contrast with previous findings in the general population, ${ }^{24}$ higher BMI was associated with lower incidence of SCD in the current study. One could speculate that the altered LV geometry and peripheral haemodynamics (ie, lower peripheral resistance) associated with increased BMI counterbalances the increased afterload from the aortic valve per se and thus reduces the risk of SCD. Our data complement similar findings in patients with severe AS. ${ }^{4}$

\section{LIMITATIONS}

The data presented are based on a retrospective analysis of a prospectively followed cohort with all its inherent limitations.

\section{Key messages}

\section{What is already known on this subject?}

- Sudden cardiac death (SCD) is a significant concern in asymptomatic patients with aortic stenosis (AS) with a reported incidence of up to 3\%/year. However, whether AS alone puts patients at risk independent of non-valve related factors, including coronary heart disease, is unclear.

\section{What might this study add?}

- The current trial demonstrates that SCD is rare $(0.4 \% /$ patientyear) in asymptomatic patients with mild to moderate AS who do not have non-valve-related risk factors. Similarly, patients who develop severe stenosis during follow-up have a low risk of SCD (0.6\%/patient-year).

\section{How might this impact on clinical practice?}

- Since the risk of sudden cardiac death in asymptomatic patients with AS is low and not primarily related to stenosis severity, alternative risk factors for SCD should actively be investigated to reduce the incidence of this serious complication.
Since LV hypertrophy regresses after AVR, it is conceivable that a reduction in risk of SCD may be obtained in the subgroup of patients with 'disproportionate' LV hypertrophy. However, the negative impact of operative mortality still needs to be considered. Patients recruited for the SEAS study were virtually free from comorbidities at baseline: on the one hand, allowing for the assessment of the impact of the valvular disease on the risk of sudden death but, on the other hand, making it difficult to generalise the current findings to the typical AS population with a high prevalence of cardiovascular disease. Moreover, the SEAS study recruited patients with mild to moderate stenosis at baseline. Despite increasing stenosis severity during follow-up, no patients reached the very severe range (jet velocity $>5.0 \mathrm{~m} / \mathrm{s}$. We, therefore, cannot entirely rule out that patients with very severe stenosis may have an increased risk of SCD due to AS alone as suggested recently. ${ }^{26}$

\section{CONCLUSION}

The risk of SCD in asymptomatic patients with AS is low and appears unrelated to stenosis severity, suggesting that interventions on the valve are unlikely to reduce the incidence of this serious complication.

Contributors All authors designed the study. AR meticulously reviewed all available documents of patients having suffered from sudden death. JM and NJ categorised patients having died from sudden cardiac death or not according to any available information and performed the statistical analysis. JM wrote the manuscript with input and critical feedback from all authors.

Funding The authors have not declared a specific grant for this research from any funding agency in the public, commercial or not-for-profit sectors.

\section{Competing interests None declared.}

Patient and public involvement Patients and/or the public were not involved in the design, conduct, reporting or dissemination plans of this research.

\section{Patient consent for publication Not required.}

Ethics approval This is a secondary analysis of the multicentre Simvastatin and Ezetimibe in Aortic Stenosis(SEAS) study (ClinicalTrials.gov number, NCT00092677). Study design and results of the SEAS study have been published previously. The study was approved by regional ethics committees in all participating countries, and all patients gave written informed consent.

Provenance and peer review Not commissioned; externally peer reviewed. Data availability statement Data are available upon reasonable request. 


\section{ORCID iDs}

Jan Minners http://orcid.org/0000-0001-7094-8348

John B Chambers http://orcid.org/0000-0002-7169-3786

\section{REFERENCES}

1 Nishimura RA, Otto CM, Bonow RO, et al. 2017 AHA/ACC focused update of the 2014 AHA/ACC guideline for the management of patients with valvular heart disease: a report of the American College of Cardiology/American heart association Task force on clinical practice guidelines. Circulation 2017;135:e1159-95.

2 Baumgartner H, Falk V, Bax JJ, et al. 2017 ESC/EACTS guidelines for the management of valvular heart disease. Eur Heart J 2017;38:2739-91.

3 Lindman BR, Clavel M-A, Mathieu P, et al. Calcific aortic stenosis. Nat Rev Dis Primers 2016;2:16006.

4 Taniguchi T, Morimoto T, Shiomi H, et al. Sudden death in patients with severe aortic stenosis: observations from the current as registry. J Am Heart Assoc 2018;7:e008397.

5 Taniguchi T, Morimoto T, Shiomi H, et al. Initial surgical versus conservative strategies in patients with asymptomatic severe aortic stenosis. J Am Coll Cardiol 2015;66:2827-38.

6 Amato MC, Moffa PJ, Werner KE, et al. Treatment decision in asymptomatic aortic valve stenosis: role of exercise testing. Heart 2001;86:381-6.

7 Otto CM, Burwash IG, Legget ME, et al. Prospective study of asymptomatic valvular aortic stenosis. Clinical, echocardiographic, and exercise predictors of outcome. Circulation 1997:95:2262-70.

8 Pellikka PA, Nishimura RA, Bailey KR, et al. The natural history of adults with asymptomatic, hemodynamically significant aortic stenosis. J Am Coll Cardiol 1990;15:1012-7.

9 Rosenhek R, Binder T, Porenta G, et al. Predictors of outcome in severe, asymptomatic aortic stenosis. N Engl J Med 2000;343:611-7.

10 Rossebø AB, Pedersen TR, Boman K, et al. Intensive lipid lowering with simvastatin and ezetimibe in aortic stenosis. N Engl J Med 2008;359:1343-56.

11 Rossebø AB, Pedersen TR, Allen C, et al. Design and baseline characteristics of the simvastatin and ezetimibe in aortic stenosis (seas) study. Am J Cardiol 2007;99:970-3.

12 Lang RM, Bierig M, Devereux RB, et al. Recommendations for chamber quantification. Eur J Echocardiogr 2006;7:79-108.
13 Baumgartner $\mathrm{H}$, Hung J, Bermejo J, et al. Echocardiographic assessment of valve stenosis: EAE/ASE recommendations for clinical practice. Eur J Echocardiogr 2009:10:1-25.

14 Deo R, Norby FL, Katz R, et al. Development and validation of a sudden cardiac death prediction model for the general population. Circulation 2016;134:806-16.

15 Hayashi M, Shimizu W, Albert CM. The spectrum of epidemiology underlying sudden cardiac death. Circ Res 2015;116:1887-906.

16 Rosenhek R, Zilberszac R, Schemper M, et al. Natural history of very severe aortic stenosis. Circulation 2010;121:151-6.

17 Pellikka PA, Sarano ME, Nishimura RA, et al. Outcome of 622 adults with asymptomatic, hemodynamically significant aortic stenosis during prolonged followup. Circulation 2005;111:3290-5.

18 Pineda AM, Kiefer TL. Asymptomatic severe aortic valve Stenosis-When to intervene: a review of the literature, current trials, and guidelines. Curr Cardiol Rep 2018:20:129.

19 Ledwoch J, Thiele H. Treatment of asymptomatic aortic valve stenosis : Watchful waiting or early intervention? Herz 2017:42:528-35.

20 Gerdts $E$, Rossebø $A B$, Pedersen TR, et al. Relation of left ventricular mass to prognosis in initially asymptomatic mild to moderate aortic valve stenosis. Circ Cardiovasc Imaging 2015;8:e003644.

21 Greve AM, Dalsgaard M, Bang CN, et al. Usefulness of the electrocardiogram in predicting cardiovascular mortality in asymptomatic adults with aortic stenosis (from the simvastatin and ezetimibe in aortic stenosis study). Am J Cardiol 2014;114:751-6.

22 Cioffi G, Faggiano P, Vizzardi E, et al. Prognostic effect of inappropriately high left ventricular mass in asymptomatic severe aortic stenosis. Heart 2011;97:301-7.

23 Duncan Al, Lowe BS, Garcia MJ, et al. Influence of concentric left ventricular remodeling on early mortality after aortic valve replacement. Ann Thorac Surg 2008:85:2030-9.

24 Cupples LA, Gagnon DR, Kannel WB. Long- and short-term risk of sudden coronary death. Circulation 1992:85:I11-18.

25 Gerdts E, Roman MJ, Palmieri V, et al. Impact of age on left ventricular hypertrophy regression during antihypertensive treatment with losartan or atenolol (the life study). J Hum Hypertens 2004;18:417-22.

26 Kang D-H, Park S-J, Lee S-A, et al. Early surgery or conservative care for asymptomatic aortic stenosis. N Engl J Med 2020;382:111-9. 